\title{
Influence of breast cancer histology on the relationship between ultrasound and pathology tumor size measurements
}

\author{
Bobbi Pritt ${ }^{1,2}$, Takamaru Ashikaga ${ }^{3,4}$, Robert G Oppenheimer ${ }^{2,5}$ and Donald L Weaver ${ }^{1,2,4}$ \\ ${ }^{1}$ Department of Pathology, University of Vermont College of Medicine, Burlington, VT, USA; ${ }^{2}$ Fletcher Allen \\ Health Care, Burlington, VT, USA; ${ }^{3}$ Departments of Biostatistics, Burlington, VT, USA; ${ }^{4}$ Vermont Cancer \\ Center; Burlington, VT, USA and ${ }^{5}$ Departments of Radiology, Burlington, VT, USA
}

\begin{abstract}
Establishing an accurate primary invasive breast cancer size is crucial for patient management. Although ultrasonographic measurement is reported to correlate reliably with the gold standard pathology measurement, few authors have examined the influence of histologic subtype on ultrasound measurement. The common subtypes of invasive breast carcinoma, ductal and lobular, have different growth patterns, which may influence the ability of ultrasound to predict pathologic size. For this analysis, ultrasound and pathology reports were retrospectively reviewed for 204 women with 210 invasive breast cancers, including 129 ductal, 41 lobular, and 40 mixed pattern ductal and lobular carcinomas. For each tumor, the largest pathology and ultrasound dimensions were compared using Pearson's correlations, linear regression, paired $t$-tests and Wilcoxon signed ranks tests, stratified by histologic subtype. The Hodges-Lehmann approach was used to obtain $95 \%$ confidence intervals $(\mathrm{Cl})$ for median difference of the sizes. Ultrasonography consistently underestimated pathologic tumor size; the overall median difference was $3.5 \mathrm{~mm}$ (Cl: $2.5-4.0 \mathrm{~mm}$ ) and for subtypes: $2.5 \mathrm{~mm}$ (Cl: 1.5-3.5 mm) for ductal pattern; $3.0 \mathrm{~mm}(\mathrm{Cl}: 1.5-4.5 \mathrm{~mm}$ ) for mixed pattern; and in contrast, $7.5 \mathrm{~mm}$ (Cl: $5.0-$ $13.5 \mathrm{~mm}$ ) for lobular pattern tumors. Significant correlations of similar magnitude, were observed between size measurements for ductal, lobular, and mixed subtypes $(r=0.816,0.811$ and 0.672 , respectively; all $P<0.001)$; however, linear regression models differed between subtypes. Although practical and widely available, ultrasonography tends to underestimate pathologic tumor size. The size difference may be large for lobular carcinomas, potentially influencing stage; differences are less pronounced for ductal and mixed subtypes. Pathologic tumor size can be estimated from the ultrasonographic measurement, particularly if the histologic tumor subtype is known. The results of this study underscore the continued benefit of pretreatment tumor histology.
\end{abstract}

Modern Pathology (2004) 17, 905-910, advance online publication, 23 April 2004; doi:10.1038/modpathol.3800138

Keywords: breast neoplasms; cancer measurement; cancer staging; comparative studies; pathology; ultrasonography

Accurate measurement of a primary invasive breast cancer is crucial for staging and patient management and is traditionally obtained by the pathologist from the surgical resection specimen. Although pathologic measurement is regarded as the gold standard, circumstances exist where it is not available or markedly altered prior to surgical excision. Neoadjuvant chemotherapy is now commonly employed, and the measurement of the tumor in the

Correspondence: Dr DL Weaver, MD, Department of Pathology, Given E-203, Health Science Complex, University of Vermont College of Medicine, Burlington, VT 05405-0068, USA.

E-mail: donald.weaver@uvm.edu

Received 13 February 2004; revised 18 March 2004; accepted 23 March 2004; published online 23 April 2004 subsequent resection often does not accurately represent the pretreatment size. Tumor measurement can be particularly challenging when largecore or vacuum-assisted needle biopsy removes part or all of a tumor prior to resection. In this situation, the pathologist may need to estimate total size based on measurements from both biopsy and resection specimens; unarguably, this is not an ideal measurement. Finally, the advent of noninvasive ablative therapies may eventually render the intact surgical resection specimen obsolete for some patients. In all of these instances, the clinician may rely on the breast imaging measurement of the tumor for staging and treatment planning, and the pathologist may need to use the imaging measurements to confirm the pathologic impression. This requires an 
understanding of the relationship between the imaging measurement and the pathology standard.

Many authors have examined the correlation of tumor size obtained by pathology and radiology, using imaging modalities such as mammography, ultrasonography, and magnetic resonance imaging. ${ }^{1-15}$ These studies indicate that although magnetic resonance imaging is accurate at predicting pathologic tumor size, ultrasonography is more widely available, relatively faster and less expensive. For these reasons, ultrasound appears to be the most useful imaging modality for preoperative tumor assessment. Several studies indicate that ultrasonography is a viable method for determining tumor size, but that actual tumor size is underestimated. ${ }^{1,3,8,11,15}$ Published studies comparing ultrasonography and pathology measurement, with their corresponding Pearson's correlation coefficients are presented in Table 1.

When determining tumor size, it is reasonable to hypothesize that histologic subtype may affect measurement. The two most commonly encountered histologic subtypes, invasive ductal carcinoma and invasive lobular carcinoma, have different patterns of invasion. While invasive lobular carcinoma tends to have a diffuse, infiltrative, poorly circumscribed growth pattern, invasive ductal carcinoma is generally circumscribed and more likely to be associated with fibrosis. The growth pattern of tumors with a mixture of ductal and lobular morphology may vary. Based on these differences, it is probable that tumors with differing histologic subtypes would have different radiographic appearances. Despite this potential, few studies have examined the specific impact of histologic subtype on ultrasonographic

Table 1 Correlation of ultrasonography and pathology measurements in reported series

\begin{tabular}{lccc}
\hline Study & Year & \# Tumors & $\begin{array}{c}\text { Correlation } \\
\text { coefficient }\end{array}$ \\
\hline Boetes et al & 1995 & 61 & $\begin{array}{c}\text { Not given } \\
\text { Davis } \text { et al }\end{array}$ \\
$\begin{array}{l}\text { Finlaysen and } \\
\text { MacDermott }\end{array}$ & 1996 & 12 & 0.45 \\
Fornage et al & 1980 & 35 & 0.70 \\
Forouhi et al & 1994 & 31 & 0.84 \\
Herrada et al & 1997 & 52 & 0.94 \\
Hieken et al & 2001 & 146 & 0.60 \\
Madjar et al & 1993 & 98 & 0.63 \\
Pain et al & 1992 & 86 & 0.75 \\
Skaane and Skjorten & 1999 & 70 & 0.69 \\
Tresserra & 1999 & 174 & 0.72 \\
Yang et al & 1997 & 39 & 0.92 \\
& & & \\
Mean of 11 published & & & 0.74 \\
studies & 2003 & 210 & 0.79 \\
Current study & & & \\
\hline
\end{tabular}

\footnotetext{
${ }^{\mathrm{a}}$ Study employed high-resolution ultrasound.

bIncludes all data for comparison purposes, not stratified by histologic subtype.
}

measurement. ${ }^{1,3,8,13}$ Of these, one group examined only invasive lobular carcinomas ${ }^{13}$ and another examined only invasive ductal carcinomas. ${ }^{3}$ The remaining two studies ${ }^{1,8}$ had limited numbers of invasive lobular carcinoma (6 and 9 respectively), compromising the ability to draw conclusions regarding the influence of histologic subtype. The current study was undertaken to further investigate the influence histology exerts on assessment of preoperative tumor size determined by ultrasound.

\section{Materials and methods}

\section{Patient Selection Criteria}

Following approval by the University of Vermont Investigational Review Board, adult female patients with invasive ductal, lobular, or mixed pattern ductal and lobular carcinoma evaluated with needle core biopsy at Fletcher Allen Health Care from 1997 to 2002 were identified. Retrospective chart review was undertaken to determine which patients had tumor measurements by both pathology and ultrasonography. For inclusion, patients must have had definitive tumor measurements from both preoperative ultrasonography and the final surgical resection specimen. The locations of the tumor on both pathology and ultrasonography reports were compared to verify that the same lesion was being measured. Patients were excluded if lesion location was not specified or if they had undergone preoperative chemotherapy or radiotherapy. Tumors other than the specified subtypes (eg mucinous, tubular) and tumors comprised primarily of carcinoma in situ were excluded from this study. The percentage of carcinoma in situ was not recorded. For more than one tumor to be included in the same patient, the tumors needed to be distinctly separate from one another. No information was gathered regarding race, ethnicity, health status, previous malignancy, tumor grade, lymph node involvement, or metastases. There were a total of 204 patients with 210 invasive breast carcinomas identified, comprised of 129 invasive ductal carcinomas, 41 invasive lobular carcinomas, and 40 invasive mixed pattern ductal and lobular carcinomas. These sample sizes provided sufficient statistical power to detect correlation coefficient values between pathology and ultrasonography measurements as low as $r=0.40$ with a sample size of $n=40$ and as low as $r=0.21$ with a sample size of $n=129$ using a $5 \%$ directional test.

\section{Ultrasonography Measurements}

Most ultrasonography for this study had been performed at the University of Vermont affiliated hospital, Fletcher Allen Health Care, Breast Care Center using Acuson 128 ultrasound machines with 7-10 MHz Linear transducers (1997-1999) and GE 
Logic 700 ultrasound machines with $7-13 \mathrm{MHz}$ Linear Matrix transducers (2000-2002). The standard protocol for sonographic evaluation of lesion size was to record the largest diameter in both the radial and anti radial planes. For analysis purposes, the maximum measurement was rounded to the nearest millimeter.

\section{Pathology Measurements}

Surgical pathology breast specimens were processed according to a standard protocol. Each breast excision or mastectomy specimen was serially sectioned and fixed in formalin overnight. The tumor was then measured in three dimensions, to the nearest millimeter, and submitted for microscopic evaluation. In general, the gross measurement of the tumor was used for staging. However, if the microscopic tumor measurement of the largest dimension was substantially greater than the largest gross measurement (eg due to microscopic tumor extension into surrounding tissues), or substantially smaller than the gross measurement (eg due to adjacent dense fibrocystic change), the microscopic measurement was utilized for staging. During the study period, histologic subtype was commonly based on morphology and pattern of invasion; e-cadherin immunohistochemical stains were not utilized to confirm lobular histology.

\section{Statistical Approach}

For each tumor, the largest dimension measured by pathology and ultrasonography was compared using Wilcoxon signed ranks tests overall and stratified by histologic subtype. The Hodges-Lehmann approach was then used to obtain 95\% confidence intervals (CI) for the median of the paired size differences. Pearson's correlation coefficients were calculated to assess the strength of the linear relationship between the two measurements along with a test of equality of correlation coefficients across tumor subtypes. Linear regression slope and intercept estimates were obtained to examine the predictive relationship between the two measurements. A 5\% significance level was used for testing purposes and 95\% confidence intervals were obtained to complement hypothesis tests. Calculations were conducted using SYSTAT (SPSS Inc., Chicago, IL, USA, version 10, 2000).

\section{Results}

Pathology and ultrasonography size measurements, along with significance levels comparing the two measures, are presented in Table 2. The mean pathology size for all tumor types was $18.8 \pm 15.2 \mathrm{~mm}$ compared to the mean ultrasound value of $14.6 \pm 9.6 \mathrm{~mm}(P<0.001)$. Mean tumor
Table 2 Pathology and ultrasound measurements by tumor histology

\begin{tabular}{lccc}
\hline Subtype & Pathology $(\mathrm{mm})$ & Ultrasound (mm) & P-value \\
\hline All $(\mathrm{n}=210)$ & & & \\
Mean \pm s.d. & $18.8 \pm 15.2$ & $14.6 \pm 9.6$ & $<0.001$ \\
Range & $0.5-140.0$ & $2.0-61.0$ & \\
Median & 15.0 & 12.0 & \\
& & & \\
Ductal (n=129) & & & \\
Mean \pm s.d. & $16.1 \pm 9.4$ & $13.6 \pm 8.0$ & $<0.001$ \\
Range & $0.5-60.0$ & $4.0-61.0$ & \\
Median & 15.0 & 12.0 & \\
& & & \\
Lobular (n=41) & & $19.2 \pm 13.7$ & $<0.001$ \\
Mean \pm s.d. & $29.6 \pm 25.7$ & $5.0-60.0$ & \\
Range & $7.0-140.0$ & 15.0 & \\
Median & 22.0 & & \\
& & $13.1 \pm 7.8$ & 0.004 \\
Mixed (n=40) & $16.5 \pm 10.9$ & $2.0-40.0$ & \\
Mean \pm s.d. & $1.2-52.0$ & 11.0 & \\
Range & 13.0 & & \\
Median & & & \\
\hline
\end{tabular}

s.d. = standard deviation;

${ }^{\mathrm{a}}$ Wilcoxon signed ranks test.

size for the invasive ductal carcinomas was $16.1 \pm 9.4 \mathrm{~mm}$ based on pathology measurement, while the mean for ultrasound was $13.6 \pm 8.0 \mathrm{~mm}$ $(P<0.001)$. The mean tumor size for the invasive lobular carcinomas was $29.6 \pm 25.7 \mathrm{~mm}$ based on pathology and $19.2 \pm 13.7 \mathrm{~mm}$ for ultrasonography $(P<0.001)$. The mean pathology and ultrasonography measurements for the invasive mixed pattern ductal and lobular carcinomas were $16.5 \pm 10.9$ and $13.1 \pm 7.8 \mathrm{~mm}(P=0.004)$, respectively.

Combining all subtypes, the median of the differences between the pathology and ultrasonography measurements was $3.5 \mathrm{~mm}$ (95\% CI: 2.5$4.0 \mathrm{~mm}$ ). For tumors of ductal subtype, the median of the differences was $2.5 \mathrm{~mm}$ (95\% CI: 1.5-3.5 mm). In contrast, the median of the differences for lobular subtype tumors was $7.5 \mathrm{~mm}$ (95\% CI: $5.0-13.5 \mathrm{~mm}$ ), while tumors with a mixed pattern had a median of the differences of $3.0 \mathrm{~mm}$ ( $95 \%$ CI: $1.5-4.5 \mathrm{~mm}$ ).

Plots of the individual pathology and ultrasound measures are presented in Figure 1 for each subtype. The linear regressions relating the pathology and ultrasound measurements in Figure 1 are summarized in Table 3. The Pearson correlation coefficients were of similar magnitude and were all statistically greater than zero $(r=0.816,0.811$ and 0.672 , respectively for invasive ductal, invasive lobular, and invasive mixed pattern ductal and lobular subtypes; all $P<0.001)$. For invasive ductal carcinomas, the estimated regression intercept of $3.212 \pm 0.941 \mathrm{~mm}$ was significantly greater than zero $(P=0.001)$, while the intercepts for invasive lobular and mixed pattern ductal and lobular carcinomas were not. Further, the linear regression slope for all tumor subtypes combined $(1.247 \pm 0.067)$ was 

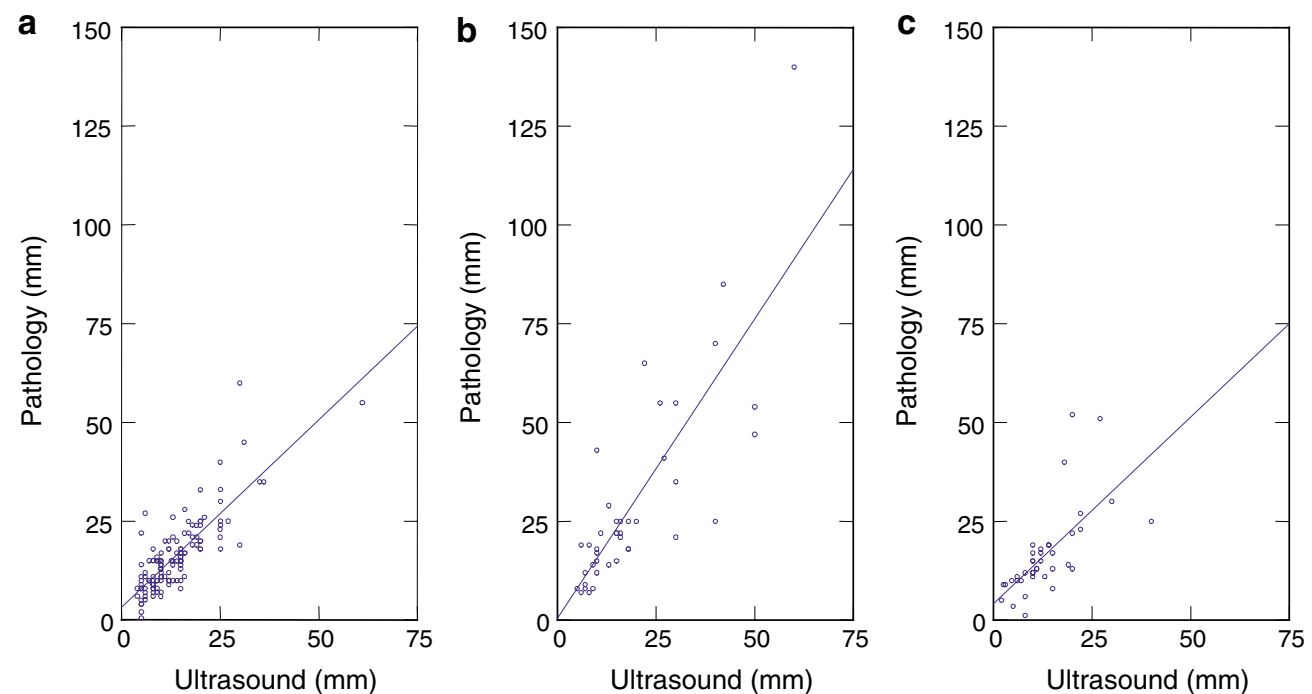

Figure 1 Comparison of maximum pathology and ultrasound measurements for ductal, lobular, and mixed pattern invasive breast carcinomas. Panel a: Invasive ductal carcinomas, $n=129$. Panel b: Invasive lobular carcinomas, $n=41$. Panel c: Mixed pattern invasive ductal and lobular carcinomas, $n=40$. Solid line indicates linear regression relationship.

Table 3 Linear regression and correlation results

\begin{tabular}{llc}
\hline Tumor histology & $\begin{array}{c}\text { Regression } \\
\text { Slope } \pm \text { s.e. (P-value) } \\
\text { Intercept } \pm \text { s.e. (P-value) }\end{array}$ & $\begin{array}{c}\text { Pearson correlation } \\
\text { (P-value) }\end{array}$ \\
\hline All $(n=210)$ & $1.247 \pm 0.067(<0.001)$ & $r=0.790(<0.001)$ \\
& $0.616 \pm 1.172(0.060)$ & \\
Ductal $(n=129)$ & $0.949 \pm 0.060(0.396)$ & $r=0.816(<0.001)$ \\
Lobular $(n=41)$ & $3.212 \pm 0.941(0.001)$ & \\
& $1.515 \pm 0.175(0.004)$ & $r=0.811(<0.001)$ \\
Mixed $(n=40)$ & $0.443 \pm 4.117(0.915)$ & \\
& $0.945 \pm 0.169(0.744)$ & $r=0.672(<0.001)$ \\
& $4.159 \pm 2.555(0.112)$ & \\
\hline
\end{tabular}

s.e. $=$ standard error; pathology size can be estimated using the linear regression function $y=m(x)+b$, where $y$ is the estimated pathology size, $m$ is the slope ${ }^{a}, x$ is the ultrasound measurement and $b$ is the intercept $\mathrm{t}^{\mathrm{b}} ; P$-values are for null hypothesis comparison of actual data to ideal slope of one and intercept of zero.

significantly greater than unity $(P<0.001)$. More importantly, the slope for the invasive lobular subtype $(1.515 \pm 0.175)$ was significantly greater than unity $(P=0.004)$ while this was not the case for the invasive ductal and mixed pattern subtypes $(P=0.396$ and 0.744 , respectively). It should also be noted that the slope for the invasive lobular carcinomas was significantly larger than the corresponding slopes for the invasive ductal $(P=0.002)$ and the mixed pattern $(P=0.019)$ subtypes.

Predicted pathology size can be calculated from the ultrasound measurement utilizing the data from the linear regression analysis and the formula $y=m(x)+b$, where $y$ is the pathology measurement, $m$ is the calculated slope of the regression line, $x$ is the ultrasound measurement, and $b$ is the intercept.

\section{Discussion}

The current study was performed to investigate specifically the influence of histologic subtype on ultrasonographic measurement. An overall good correlation between pathology and ultrasonography measurements was predicted based on previous studies and confirmed in the current study (Table 1); however, due to the more diffuse, less circumscribed nature of lobular carcinoma, a larger difference between pathology and ultrasonography measures and a poorer linear regression relationship between the two approaches was expected and confirmed for tumors exhibiting a lobular growth pattern.

Although ultrasonography is a practical, widely available and commonly used modality for preoperative tumor assessment, it tends to underestimate actual tumor size for all tumor subtypes studied by about $3.5 \mathrm{~mm}$. The underestimate is most pronounced for lobular tumors where the median difference between the pathology gold standard and ultrasonography may be as small as $5.0 \mathrm{~mm}$ or as large as $13.5 \mathrm{~mm}$ based on the $95 \%$ CI potentially influencing stage and treatment. While the differences between the two methods were less pronounced for ductal and mixed pattern subtypes, there was still a significant median underestimation of 2.5 and $3.0 \mathrm{~mm}$ observed, respectively.

Several observations can be made on review of the linear regression and correlation results presented in Table 3. If ultrasound measurements perfectly predicted pathology measurements, the Pearson correlation coefficient should be very close to 1.0, and the linear regression relationship between the two measures should have an intercept equal to zero and a slope equal to unity. Correlation values of 
$r=0.816$ (ductal), $r=0.811$ (lobular) and $r=0.672$ (mixed pattern) and the corresponding 95\% CIs ([0.741-0.891], [0.685-0.937] and [0.543-0.801], respectively), indicate some overlap in these correlational values. The linear regression results also differed between tumor subtypes. The regression intercept of $3.212 \pm 0.941 \mathrm{~mm}$ for invasive ductal carcinoma was significantly greater than zero $(P=0.001)$ and the slope estimate was not significantly different from unity $(P=0.396)$ indicating that ultrasonography measurements were consistently underestimating pathology measurements by $3.2 \mathrm{~mm}$ across all invasive ductal carcinoma tumor sizes. The intercept for invasive lobular carcinoma did not differ from zero $(P=0.915)$ although the slope estimate of $1.515 \pm 0.175 \mathrm{~mm}$ was significantly greater than unity $(P<0.001)$. This indicated that ultrasound did not underestimate size for smaller invasive lobular carcinomas but that ultrasonography became more conservative as tumor size enlarged. In contrast to invasive ductal and invasive lobular subtypes, the linear regression intercept of $4.159 \pm 2.555 \mathrm{~mm}$ for the mixed pattern ductal and lobular carcinomas did not differ significantly from zero $(P=0.112)$, and the slope estimate did not differ from unity $(P=0.744)$. However, some caution should be exercised here since the mixed pattern subtype also had the weakest regression relationship with a Pearson correlation coefficient of $r=0.672$.

These observed differences between the ultrasonography and pathology measures, and the varied linear regression relationships between these two measures across all tumor subtypes underscore the continued need for tumor histology, regardless of whether or not a complete surgical excision is performed. Size of invasive tumor commonly guides treatment and patients with a tumor greater than $1.0 \mathrm{~cm}$ are typically offered adjuvant chemotherapy. On the other hand, it is less common for patients with node negative disease and a tumor less than $1.0 \mathrm{~cm}$ to undergo adjuvant chemotherapy. When pathologic tumor size is not available, knowledge of the histologic subtype can allow estimation of pathologic size from the ultrasonographic measurement, or in the case of lobular carcinoma, alert the clinician to a possible significant underestimate of tumor size. Relying on ultrasonography alone may underestimate actual tumor size and potentially impact patient management. For example, as can be observed from Figure 1, a patient with a $15 \mathrm{~mm}$ lesion by ultrasound would be predicted to have a pathologic lesion measuring $17.4 \mathrm{~mm}$ for invasive ductal carcinoma, $18.3 \mathrm{~mm}$ for a mixed pattern invasive carcinoma, and $23.2 \mathrm{~mm}$ for invasive lobular carcinoma. Note that in the case of lobular carcinoma, the ultrasound size would place the tumor in the T1c category, while the predicted measurement would result in a T2 classification. ${ }^{16}$

Although this study has clarified the relationship between tumor histology, ultrasound measurements, and pathology measurements, it has several limita- tions. First, it is important to note that the formulas presented to predict pathology size are most accurate for invasive ductal pattern carcinomas. While patient sample sizes were determined by and are sufficient for statistical analysis and comparison of the differing relationships for the three patterns of invasive carcinoma evaluated, prediction models were not the primary objective of the study; larger patient cohorts for invasive lobular and mixed pattern ductal and lobular carcinoma would be needed to develop prediction formulas with greater predictive utility. Second, patients with large tumors may have been under-represented because patients treated with preoperative chemotherapy were excluded from the study. Finally, the amount of concurrent carcinoma in situ was not noted for each tumor. This should not influence the results of this study since carcinoma in situ would be included in the overall pathologic measurement of the tumor if it comprised part of the mass-forming lesion and, contrary to the study findings, any contribution of adjacent in situ carcinoma to ultrasound size would have overestimated pathologic size. Tumors that were comprised primarily of carcinoma in situ were excluded from the study.

In conclusion, ultrasonography, a common method for assessing clinical breast tumor size, does underestimate pathologic measurements. Histologic subtype influences the accuracy of the ultrasound measurement with the greatest differences between ultrasound and pathology measurements observed in lobular carcinomas. Knowledge of the histologic subtype can improve predicted pathology size estimates in cases where pathology measurements are unavailable or have been compromised by prior biopsy or therapy.

\section{Acknowledgements}

This work was supported by a grant (P30-CA22435) from the National Cancer Institute. The views expressed in this article are solely those of the authors and do not necessarily represent the official views of the National Cancer Institute, or the federal government. The data were presented in part at the United States and Canadian Academy of Pathology 92nd Annual Meeting, March 24, 2003, Washington, DC.

\section{References}

1 Boetes C, Mus RD, Holland R, et al. Breast tumors: comparative accuracy of MR imaging relative to mammography and US for determining extent. Radiology 1995;197:743-747.

2 Davis PL, Staiger MJ, Harris KB, et al. Breast cancer measurements with magnetic resonance imaging, ultrasonography, and mammography. Breast Cancer Res Treat 1996;37:1-9. 
3 Finlayson CA, MacDermott TA. Ultrasonography can estimate the pathologic size of infiltrating ductal carcinoma. Arch Surg 2000;135:158-159.

4 Fornage BD, Toubas O, Morel M. Clinical, mammographic and sonographic determination of preoperative breast cancer size. Cancer 1987;69:765-771.

5 Forouhi P, Walsh JS, Anderson TJ, et al. Ultrasonography as a method of measuring breast tumour size and monitoring response to primary systemic treatment. Br J Surg 1994;81:223-225.

6 Harms SE, Flamig DP, Hesley KL, et al. MR imaging of the breast with rotating delivery of excitation of resonance: clinical experience with pathologic correlation. Radiology 1993;187:493.

7 Herrada J, Revathy BI, Atkinson EN, et al. Relative value of physical examination, mammography, and breast sonography in evaluating the size of the primary tumor and regional lymph node metastases in women receiving neoadjuvant chemotherapy for locally advanced breast carcinoma. Clin Cancer Res 1997; 3:1565-1569.

8 Hieken TJ, Harrison J, Herreros J, et al. Correlating sonography, mammography, and pathology in the assessment of breast cancer size. Am J Surg 2000;182: 351-354.

9 Madjar H, Ladner HA, Sauerbrei W, et al. Preoperative staging of breast cancer by palpation, mammography and high resolution ultrasonography. Ultrasonogr Obstet Gynecol 1993;3:185.

10 Mutaz H, Hall-Craggs MA, Davidson T, et al. Staging of symptomatic primary breast cancer with MR imaging. AJR 1997;169:417-424.

11 Pain JA, Ebbs SR, Hern RPA, et al. Assessment of breast cancer size: a comparison of methods. Eur J Surg Oncol 1992;18:44-48.

12 Rodenko GN, Harms SE, Pruneda JM, et al. MR imaging in the management before surgery of lobular carcinoma of the breast: correlation with pathology. AJR Am J Roentgenol 1996;167:1415-1419.

13 Skaane P, Skjorten F. Ultrasonographic evaluation of invasive lobular carcinoma. Acta Radiol 1999;40: 369-375.

14 Tresserra F, Feu J, Grases PJ, et al. Assessment of breast cancer size: sonographic and pathologic correlation. J Clin Ultrasonogr 1999;27:485-491.

15 Yang WT, Lam WWM, Cheung H, et al. Sonographic, magnetic resonance imaging, and mammographic assessments of preoperative size of breast cancer. J Ultrasonography Med 1997;16:791-797.

16 Greene FL, Page DL, Fleming ID, Fritz AG, Balch CH, Haller DG, Morrow M (eds). American Joint Committee on Cancer (AJCC) Cancer Staging Manual, 6th edn. Springer: New York, 2002, pp 223-240. 